\title{
Early diagnosis with alternative approaches: innovation in lung cancer care
}

\author{
Ahmed Hasnain Jalal ${ }^{1}$, Amit Kumar Sikder ${ }^{2}$, Fahmida Alam², Sharraf Samin ${ }^{3}$, Sharmin S. Rahman \\ Md. Morshed A. Khan ${ }^{5}$, Masudur R. Siddiquee ${ }^{2}$ \\ ${ }^{1}$ Department of Electrical Engineering, University of North Florida, Jacksonville, Florida 32224, USA; ${ }^{2}$ Department of Electrical and Computer \\ Engineering, Florida International University, Miami, Florida 33174, USA; ${ }^{3}$ Department of Public Health and Informatics, Bangabandhu Sheikh \\ Mujib Medical University (BSMMU), Dhaka, Bangladesh; ${ }^{4}$ Department of Pathology, Tairunnessa Memorial Medical College, Gazipur, Bangladesh; \\ ${ }^{5}$ Department of Public Health, West Chester University of Pennsylvania, West Chester, PA, USA \\ Contributions: (I) Conception and design: AH Jalal, AK Sikder, F Alam; (II) Administrative support: None; (III) Provision of study materials or \\ patients: None; (IV) Collection and assembly of data: None; (V) Data analysis and interpretation: None; (VI) Manuscript writing: All authors; (VII) \\ Final approval of manuscript: All authors. \\ Correspondence to: Ahmed Hasnain Jalal. Department of Electrical Engineering, University of North Florida, Jacksonville, Florida 32224, USA. \\ Email: ajala003@fiu.edu.
}

\begin{abstract}
Cancer is one of the primary concerns of mortality throughout the world in the present day. Among different types of cancers, lung cancer (LC) is predominant (21.77\% of overall cancer caused death). It is classified into two major categories: oat cell or small cell lung cancer (SCLC) and non-small cell lung cancer (NSCLC). The mortality due to NSCLC $(\sim 85 \%)$ is almost six times higher than the SCLC $(15 \%)$. The identification of risk factors can reduce cancer burden and human fatality; however, this cannot prevent the severity of LC. In this case, the early diagnosis of LC can be an effective pathway in providing better treatment to the patients, which eventually may result in less fatality. This article offers an overview of several existing screening methods for the early diagnosis of LC. Nevertheless, these methodologies have limitations of reliability towards the diagnosis of cancer malignancy. Therefore, the prospects of different alternative approaches and their challenges to overcome this barrier for the early diagnosis of malignant tumor cells have been articulated in this article.
\end{abstract}

Keywords: Lung cancer (LC); early detection; alternative approaches

Received: 01 December 2019; Accepted: 25 February 2020; Published: 10 January 2021.

doi: $10.21037 /$ shc. 2020.03 .03

View this article at: http://dx.doi.org/10.21037/shc.2020.03.03

\section{Introduction}

Over the past few years, cancer has been the root cause of disease mortality in the world. Among different variants of cancer, lung cancer (LC) is the most dangerous and leading category of cancer worldwide $(1,2)$. According to the World Health Organization, approximately 2.09 million people suffered from this disease, and it was caused around 1.76 million of mortality among them in the year 2018 (1). LC originates from the alteration of healthy cells into malignant cells in several biochemical processes that typically grow from pre-cancerous lesions to nonbenign tumors, lumps, and neoplasms. This feature, with the fast formation of abnormal cells, raises outside their typical borders. These cancer cells later may invade adjacent organs of the body from the lungs and spread over other parts of the body. This invasion refers to metastasizing, which promotes fatalities from cancer. LC is predominantly caused by two major clusters of histological patterns (2): oat cell or small cell lung cancer (SCLC) and non-small cell lung cancer (NSCLC). 80-85\% of LC are NSCLC, whereas the rest of the $10-15 \%$ are oat cell type (2). NSCLC is subcategorized in several types following their histological patterns, such as adenocarcinoma, squamous cell carcinoma, large cell 
carcinoma, adenosquamous carcinoma, and sarcomatoid carcinoma $(3,4)$. The roots of adenocarcinoma and squamous cell carcinoma are typically formed in mucous, whereas large cell carcinoma origins in the squamous cells. However, large cell carcinoma intends to spread more rapidly compared to other types of NSCLC due to its aggressive histological patterns. Adenosquamous carcinoma and sarcomatoid carcinoma are fewer common subspecies of LC. Typically, tobacco smoking is the primary cause (80-90\%) of promoting all these patterns of LC and the cause of $80-90 \%$ of deaths per year (5). Carcinoid tumors, lymphomas, adenoid cystic carcinomas, sarcomas, or rooted from other organs (e.g., breast, pancreas, kidney, etc.) cause fewer than $5 \%$ of malignancy in lung cells (6).

The four following significant intrinsic and extrinsic factors have been identified, causing LC so far by the International Agency for Research on Cancer (IARC) (7): (I) a person's genetic influences, (II) physical carcinogens such as ionizing and ultraviolet (UV) radiation, (III) toxic chemical (e.g., tobacco, a food toxin, asbestos, and drinking water contaminants (e.g., arsenic); and (IV) organic carcinogens, such as infections from specific microorganisms (e.g., viruses, bacteria, or parasites). Apart from these, aging leads to the risk of cancers as the cellular reparation mechanisms get inactive with the increment of oldness. Besides, the ingestion of alcohol and tobacco smoke, unhealthy diet, inactive lifestyle, and cancer-causing chronic infections due to Hepatitis B and C viruses, Human papillomavirus (HPV), Helicobacter pylori, and EpsteinBarr virus are the key danger factors to activate LC (8-11).

LC can be prevented by following a healthy lifestyle such as alcohol and tobacco avoidance, a healthy diet, and physical activities (12). Also, the regular screening of lung can reduce the severity of LC. For instance, the results of CT and X-ray in an earlier screening test for the stage I diagnosis of LC was positive for the $63 \%$ and $47.6 \%$, respectively (13). According to the report of the National Lung Screening Trial (NLST), the mortality rate of LC reduced by $20 \%$ due to early screening (13). Therefore, early diagnosis not only provides a pathway for the adequate treatment to stop further progression of carcinogenic cells but also it is a potential solution to prevent LC casualties.

The existing methodologies of LC screening include $\mathrm{X}$-ray and computed tomography (CT). However, the reliability of these techniques is a concern as the false positive rate was over $15 \%$ among these studies (14). Hence, in recent years, several alternative approaches (e.g., metabolomic, transcriptomic, genomic, and proteomic) for the identification of cancerous biomarkers have been explored for the early detection of LC so far. These approaches have been achieved through different pathological, molecular, and biochemical analyses, such as biopsy (supported by different methods: e.g., ctDNA, circulating tumor cells), sputum analysis, bronchoscopy, confocal micro-endoscopy, biomolecular markers (volatile, antibodies, etc.), endobronchial ultrasonography, optical coherence tomography, and positron emission tomography. This article demonstrates the potential and reliable pathway towards the early diagnosis of LC through these methodologies.

\section{Current approaches for LC diagnosis}

The two major diagnosis approaches to LC are (I) $\mathrm{X}$-ray, and (II) computed tomography (CT) scan. Their methodologies, contributions, and limitations have been elucidated below.

\section{$X-r a y$}

$\mathrm{X}$-ray is one of the trivial methods for early LC detection. For LC detection, a chest X-ray is used, which includes an X-ray image of the heart, lungs, blood vessels, and bones (15). During the chest X-ray, a small amount of radiation beam passes through the body of the patient, and a black-and-white image is created on a film or computer (Figure 1A). Any unusual mass or nodules become visible on the $\mathrm{X}$-ray image as the transmission of radiation varies due to the density of the cell. The main feature used in the $\mathrm{X}$-ray image is the degree of contrast and the size of the nodules. However, in the early stage of LC, the nodules developed in the lungs are relatively small and show up as low-contrast white circular dots in the X-ray plates making the detection relatively hard (16). Again, the chest X-ray contains blood vessels and rib areas that overlap with the cancer mass or nodules, making them invisible in the X-ray image (17). Thus, $\mathrm{X}$-ray is proved to be ineffective in early LC detection. Several recent studies have also investigated the effectiveness of X-ray in early LC detection. Rota et al. surveyed 765 subjects affected by LC (18). In their research, they found that the accuracy of $\mathrm{X}$-ray based detection is ten times lower than other early-stage cancer detection techniques such as tomography. Gohagan et al. conducted another study to observe the effectiveness of $\mathrm{X}$-ray in detecting different types of LC (19). The researchers found that out of 16 stage III-IV cancers, chest X-ray could only 

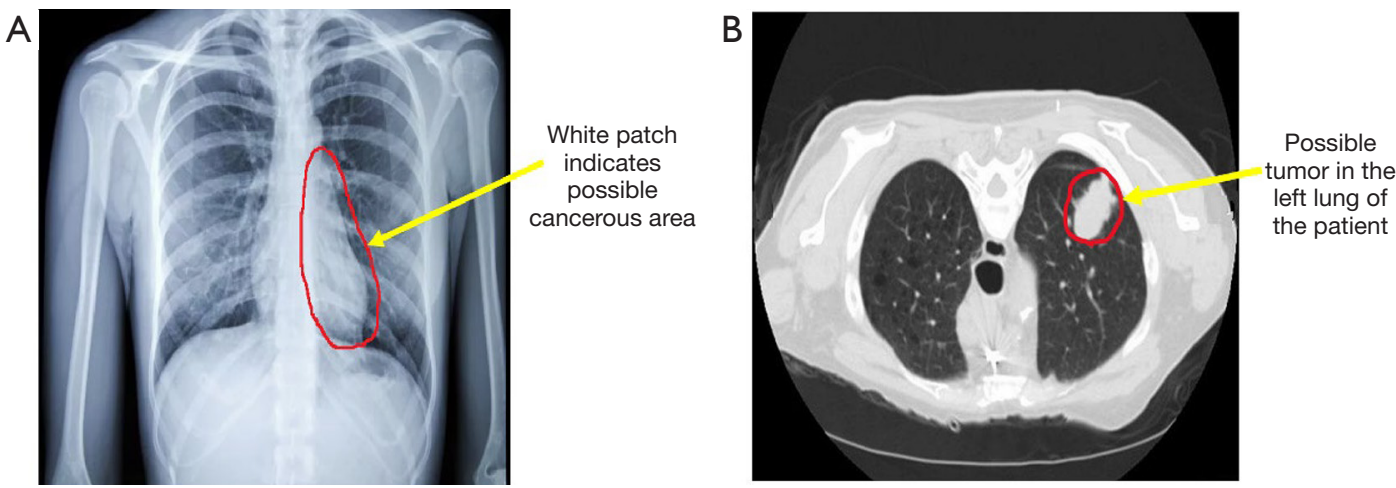

Figure 1 Current approaches for lung cancer (LC) detection (A) using chest X-ray (B) using a CT-scan.

detect nine cases. However, using deep learning methods, several research studies have been conducted to improve the accuracy of early LC detection using X-ray. Gordienko et al. presented that it is possible to remove the overlapping bone images from the $\mathrm{X}$-ray image to improve the accuracy of detecting unusual mass and nodules in the chest $\mathrm{X}$-ray using deep learning (20).

\section{CT scan}

Computed Tomography, also known as CT or CAT scan, is a specialized diagnostic method that uses a unique form of $\mathrm{X}$-ray and computer technology to create medical images for soft tissues, bones, and internal organs (21). CT scan provides more detail information than traditional chest $\mathrm{X}$-ray regarding early diagnosis of LC. CT scan produces cross-sectional images of the soft tissues in the chest and lungs area, which can be used to observe any unusual mass form or nodules (Figure 1B). Several researchers have demonstrated the efficiency of CT scans in detecting earlystage LC in their studies. Saghir et al. studied the CT scan of 4,104 smokers diagnosed with early LC and achieved an $83 \%$ detection rate (22). In another study, a total of 31,567 persons with the risk of LC have been diagnosed using a CT scan, and 484 participants have been diagnosed with cancer with a confidence rate of $95 \%$ (23). However, low-dose spiral CT scan images show pulmonary nodules, which is common among many people. This increases the chance of false-negative cases in the early detection of LC. Also, a high dose of radiation and high cost make CT scan less effective in early diagnosis (24). Recently, several new approaches have been accumulated with the traditional CT scan method to improve the accuracy of LC detection. Makaju et al. proposed a new approach to integrate image processing and machine learning technique to improve the accuracy of LC detection from CT scan images (25). In recent years, optical coherence tomography (OCT) is widely used, which is a special imaging technique. OCT uses lowcoherence light to capture higher spatial resolution than $\mathrm{CT}$ and provides high-definition cross-sectional images of inner lungs, including the airway luminal wall. This can be used to detect any lesions in the bronchial, which can be considered as a sign of LC. Nonetheless, an alternative approach is needed to minimize the use of radiation and high cost in early-stage LC detection.

\section{Alternative approaches for LC diagnosis}

\section{Biopsy}

Lung biopsy is a test done to remove a tissue sample from the lung for examination. This small piece of the lung is taken with a special biopsy needle or during surgery. This type of biopsy is called a tissue biopsy, which is performed to detect different lung diseases, including LC. There are different types of biopsies available to detect lung diseases. For example, a needle biopsy (shown in Figure 2) is performed by putting a needle into the lung to take out a sample. Transbronchial biopsy is done with a bronchoscope, a long, thin tube with a tiny camera. Video-assisted thoracoscopic surgery (VATS) uses a special tiny camera called a thoracoscope, which transmits the chest image to the computer monitor. This is a minimally invasive process where the thoracoscope is put inside the chest cavity through a small incision. Contrarily, open biopsy involves a larger incision in the patient's skin to reach and remove a small piece of the lung for further examination. These tissue biopsies have been widely used for LC diagnosis, but 


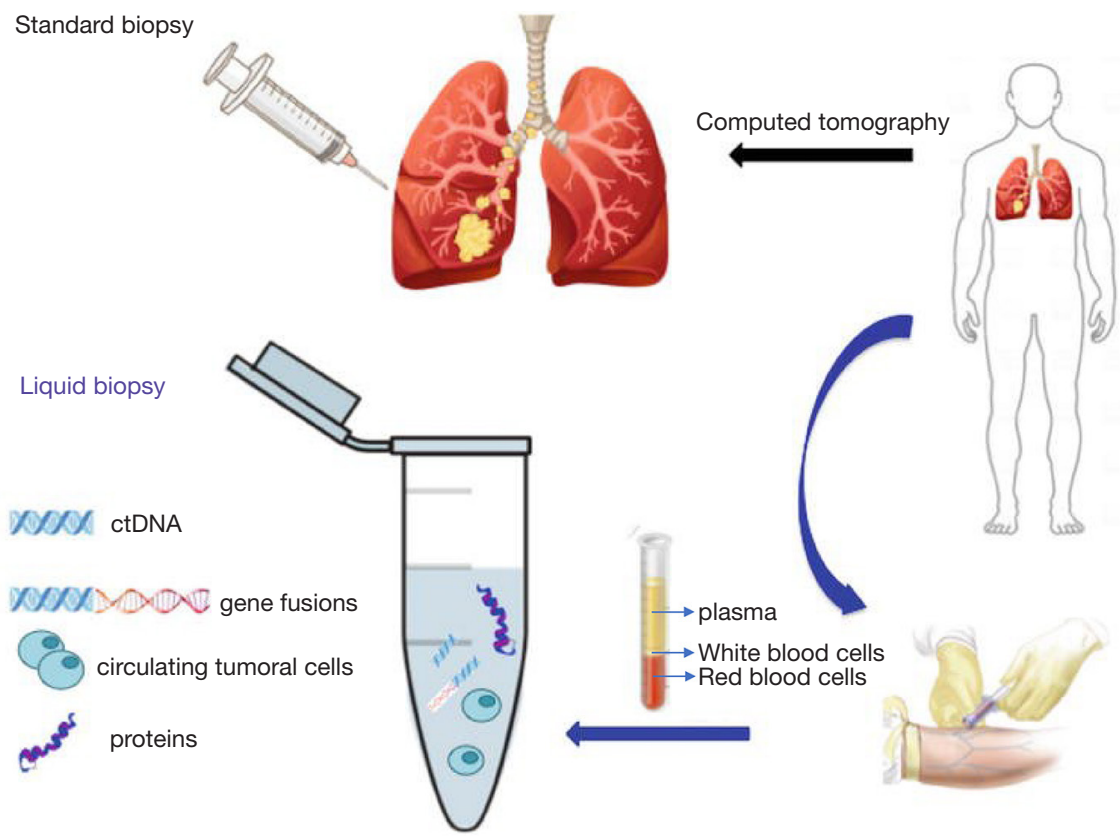

Figure 2 The examination methodology of liquid biopsy (26) * ${ }^{\star}$ (2019) (Giulio Tarro, Moreno Paolini and Alessandra Rossi). Originally published in (Licensee IntechOpen) under [the terms of the Creative Commons Attribution License (http://creativecommons.org/licenses/ by/3.0)] license. Available from: 10.5772/intechopen.85334.

the invasive nature limits their application, especially when repeated biopsies are needed. However, for early diagnosing LC, a minimally invasive procedure-liquid biopsy—aiming to primarily analyze circulating tumor cells (CTCs) and/ or circulating tumor DNA (ct DNA) has gained interest from oncologists and basic researchers (shown in Figure 2). Nowadays, liquid biopsy is more feasible in clinical practice than before, because liquid biopsy is used to predict prognosis according to genetic alterations and monitor disease based on signature molecular markers in addition to the diagnosis of LC (27).

Traditionally, the histopathologic diagnosis of LC has been made based upon information obtained from tissue biopsies. A tissue biopsy can also help oncologists for the staging of LC; therefore, treatment modalities can be determined. However, tissue biopsy has the following limitations: (I) an invasive procedure, (II) an unreliable diagnostic technique in terms of tumor heterogeneity and metastasis at distant sites, and (III) lack of repeatability. These characteristics of tissue samples can demand further investigations on the patients. On the contrary, liquid biopsy has been demonstrated to be a viable surrogate for noninvasive assessment of tumor-specific biomarkers while diagnosing LC. Therefore, it can be potentially used for a variety of clinical and investigational applications (28). Several liquid biopsies (circulating biomarkers) can be investigated in blood, for example circulating cell-free tumor DNA (cf DNA), cell-free RNA (cf RNA), exosomes, tumor-educated platelets (TEP), and circulating tumor cells (CTCs) to make early detection of LC (28). However, identifying CTC and ct DNA for LC diagnoses are limited by the low concentrations found in blood at the early stages. However, the ability to study the genomic profile of cancer cells through the noninvasive sampling of blood or other body fluids represents one of the most exciting and rapidly improving fields in diagnosing LC (29). CTCs originate by cell detachment from the primary LC into circulation. Moreover, CTCs are considered to contribute to cancer progression and the development of metastases. CTCs have been isolated in blood of patients with $\mathrm{LC}$ at variable concentrations depending on cancer types and stages of diseases. Various studies have demonstrated a potential role of CTCs as prognostic biomarkers or in predicting and monitoring response to different treatments, including targeted therapies. Compared to ct DNA, CTCs allow comprehensive studies at the DNA, RNA, and protein levels as well as functional studies, including the establishment of cell lines and xenografts (29). 


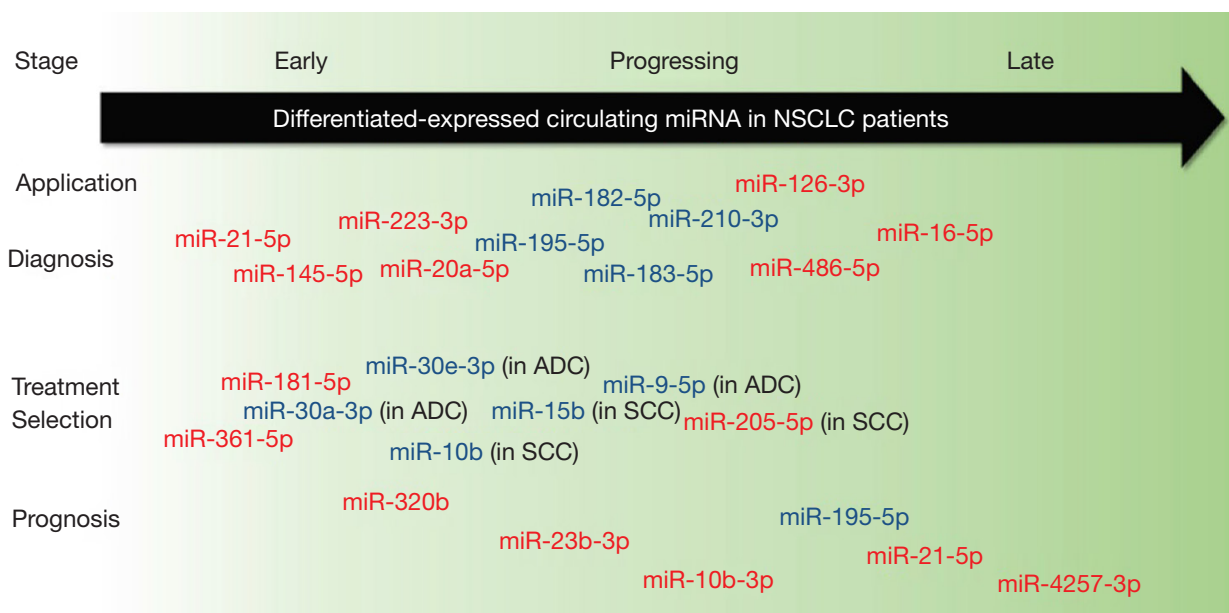

Figure 3 Potential clinical application of circulating miRNAs as tumor biomarkers for non-small cell lung cancer (NSCLC).

\section{Circulating microRNAs for LC screening}

MicroRNAs are a group of single-stranded, noncoding small RNA (length of 22 nucleotides) (30,31), which affect the stability and rate of translation of their target messenger RNA (mRNA), thus regulate gene expression $(30,32)$. There has been a significant alteration of patterns of miRNAs expressions during different developmental stages or in pathological conditions like cancer, cardiovascular diseases, etc. (33-35). Alteration of expression of miRNAs can be identified by Polymerase Chain Reaction (PCR), Northern Blotting method, microarray and deep sequencing method and can be applied for medical applications as well $(31,36,37)$.

Abnormal levels of specific miRNAs can be identified in different stages, including early-stage, progression, or metastatic stages of cancer (31), and thus, these circulating small RNAs in plasma or serum can act as significant noninvasive biomarkers for diagnosis of LC in early stages (38). Wang et al. showed that differential expressions of miRNAs have been identified in various stages like diagnosis, treatment stage, and prognosis of NSCLC (Figure 3). The red color indicates increased expression of miRNAs in the peripheral blood of NSCLC patients, where blue color denotes the decreased expressions of miRNAs (31).

Yu et al. conducted a systematic review providing a summary of using circulating miRNAs as biomarkers for LC detection and indicated the diagnostic performance of miRNAs and miRNA panels in different cases (32). Yu et al. reviewed 17 studies in total, among which individual miRNA and miRNA panels were utilized as diagnostic biomarkers for both histological subtypes and stage-specific analysis.
Considering the comparative analysis of sensitivity and specificity of both miRNA panels and individual miRNAs, the prior one appears better. However, no histology-specific miRNA could be identified because of limited changes in miRNA expressions in different histological subtypes. In the case of stage-specific analysis, both miRNA panels and individual miRNAs showed better efficacy for an advanced stage of LC diagnosis compared to early-stage with a minor difference in differential expressions of miRNAs in various stages. Boeri et al. conducted a prospective evaluation for early diagnosis of LC by CT screening trial and identified a panel of 15 miRNAs for predicting LC incidence in 2 years with sensitivity and specificity of $80 \%$ and $90 \%$, respectively (39). Sozzi et al. conducted a similar study for prospective evaluation and validated a panel of 24 miRNAs, including the 15 miRNAs identified by Boeri et al. with similar sensitivity and specificity (40). Zaporozhchenko et al. [2016] identified a panel of miR-19b and miR-183 in plasma for histology variant LC detection with both sensitivity and specificity of $95 \%$ (41). Shen et al. also used a panel of miR-21, miR-486-5p, miR-126, and miR-210 for diagnosis of adenocarcinoma yielding $92 \%$ sensitivity and $97 \%$ specificity (42). While several miRNAs (e.g., miRNA-21, miRNA-155, etc.) are frequently reported in different studies, the higher frequency does not prove the better efficacy as a diagnostic marker because of their low median specificity. However, there is always a chance affecting the identification of miRNAs by disease conditions like COPD, asthma, tuberculosis, natural aging, gender, ethnicity, smoking behavior, etc. (43-47). Moreover, sample preparation, hemolysis of samples, and variation of 


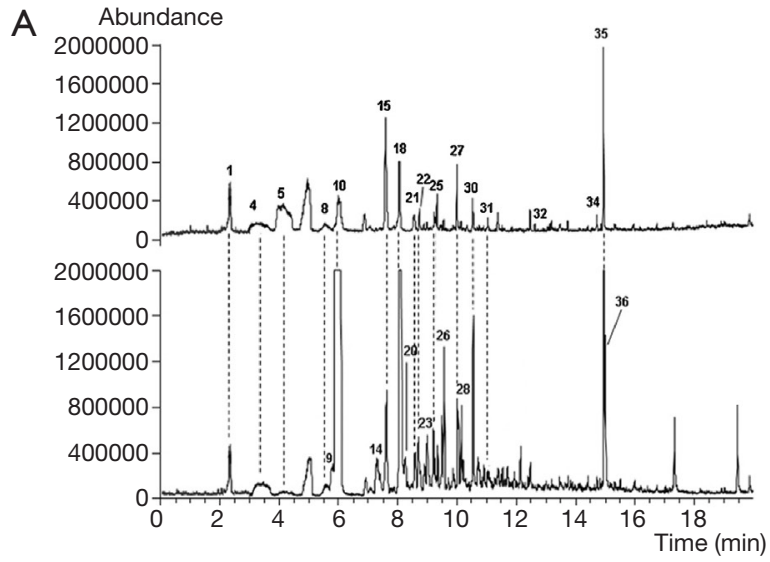

B

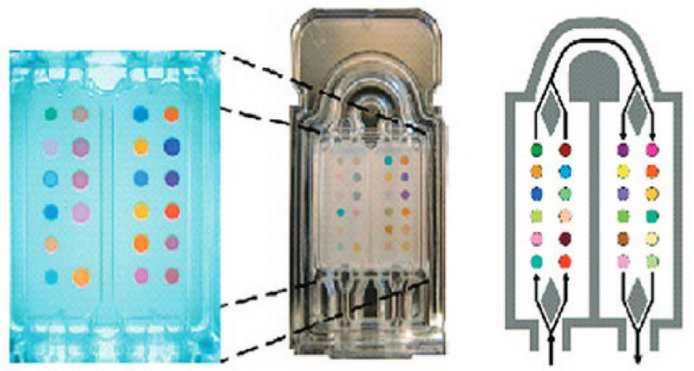

Figure 4 Volatile organic compounds (VOCs) as biomarkers for lung cancer diagnosis (A) GC/MS ion chromatograms of VOCs in benign (top) and cancerous (bottom) pleural samples, (B) 24 chemically reactive colorants used in colorimetric sensor for the detection of lung cancer from breath.

concentration of plasma and serum also affect the findings of miRNAs to a great extent (48-50). The most pragmatic approach is a combination of multiple individual miRNAs as a panel, which will enhance the diagnostic accuracy of circulating miRNAs for LC detection.

\section{Sputum analysis}

Sputum cytology is considered as the classic non-invasive screening method for assessing malignant and nonmalignant diseases (51). Although it is quite a decent method with cost-effectiveness, it showed poor sensitivity and specificity for LC screening $(52,53)$. In the early 1970s, NIH funded three randomized controlled trials that were conducted by the Mayo Clinic (54), Johns Hopkins University (55), and Memorial Sloan-Kettering (56) showing no benefit of sputum cytology as an effective outcome for the screening of LCs. Sputum cytology is not performed nowadays for early LC detection.

However, some recent studies showed that aberrant methylation of some genes persisting in the sputum of LC patients could be detectable by DNA methylation, avoiding the necessity of invasive screening procedures (57). Genetic alterations and epigenetic modifications are the earliest features of the pathogenesis of LC and DNA methylation, being one of the primeval epigenetic changes are associated with the manifestation and progress of LC (58). Liu et al. conducted a meta-analysis showing a mid-level accuracy of detection of methylated genes in sputum samples for early screening of LC with a sensitivity of 0.46 and specificity of 0.83 and they suggested to pick the superior genes as diagnostic biomarkers for LC (59). Since single DNA methylation has less efficacy as a biomarker for the detection of LC, some studies recommended combined methylated genes for improving the diagnostic value $(60,61)$. Methylated FAM19A4 showed high sensitivity and methylated RASSF1A, FHIT, MGMT, and p16 showed a high positive likelihood ratio (PLR) suggesting that they have the potential of being a diagnostic marker for the screening test (62). Also, the methylation of FAM19A4, FHIT, and MGMT were reported to play the roles in the occurrence and deterioration of LC (63-66). Therefore, methylated SOX17, CDO1, ZFP42, TAC1, FAM19A4, FHIT, MGMT, p16, and RASSF1A are useful in the screening and auxiliary detection of LC.

\section{Detection of volatile organic compounds (VOCs) as biomarkers}

The differences between the levels of VOCs in different biofluids of a healthy person and cancer ailed patients can be a potential pathway to determine the existence and severity of LC. The major sources of these olfactory biomarkers are several biofluids, such as blood, plasma, interstitial fluid, exhaled breath, and urine. Horvath et al. suggest aroma(s) in the blood can be an appropriate screening approach for the detection of LC (67). Matsumura et al. demonstrated an ex-vivo analysis of urinary VOCs as biomarkers for LC (68). Liu et al. (shown in Figure 4A) found three key organic groups (ketones, alcohols, and benzene) in pleural samples where their derivatives were promising biomarkers 
for cancer malignancy. Among $76 \mathrm{VOCs}$, nine of them including ethyl acetate, dichloromethane, n-heptane, xylene, ethyl-benzene, cyclo-hexanone, cyclo-hexanol, tetra-methyl benzene, and 2-ethyl-1-hexanol, are plausible compounds for screening to distinguish nonbenign from malignant pleural effusions. Due to apoptosis and necrosis, dead LC cells also origins of some specific VOCs, such as 1,3-ditert-butylbenzene, 2,6-di-tertbutyl-1,4-benzoquinone, and n-decane (69).

Several analytical methods, such as GC-MS, GS-MS techniques with olfactometer (GS-MS-O), proton transferreaction MS (PTR-MS), selected ion flow tube MS (SIFTMS), laser spectrometry and ion mobility spectrometry (IMS), electron impact mass spectroscopy (EIMS) integrated with two-dimensional gas chromatography $(\mathrm{GC} \times \mathrm{GC})$ were employed for the detection of $\mathrm{VOC}$ biomarkers from urinary fluids, blood, plasma or interstitial fluids (70-74). However, e-noses are being widespread for their real-time and noninvasive detection from breath. Such form factors for chemi-resistive, optical, and electrochemical sensing approaches have been achieved for the miniaturization of e-noses (75-78). Obermeier demonstrated integrated e-noses with multiple amperometric sensors for the detection of VOC biomarkers, such as aldehyde, NO, and $\mathrm{CO}$ at sub-ppb levels for the diagnosis of LC (79). Mazzone's group from Cleveland Clinic verified colorimetric based e-noses combined with a prediction model to diagnose LC, shown in Figure $4 B(80)$. In their study, they collected 229 subjects' data and diagnosed 92 individuals as carcinogenic patients with a diagnostic accuracy of $0.8 \mathrm{C}$-statistics. They also explored chemi-resistive based e-noses for the diagnosis of LC from respiration (81). They applied multivariate analysis methods for the sorting of benignity and malignancy of lung cells. Further, the support vector machine (SVM) was employed to generate an estimated model from the data they obtained. Their e-noses showed a sensitivity of $71.4 \%$ and $91.9 \%$ of specificity for the diagnosis of LC.

\section{Antibody-based biomarkers for early LC diagnosis}

Screening trials employing existing technologies, such as chest $\mathrm{x}$-ray, CT, or sputum analysis, have far been incapable of reducing the mortality caused by LC. Most of these techniques suffer from inaccuracy and lower reliability due to the wrong diagnosis by false-positive errors caused by infection, benign tumors, pregnancy, and other factors (14). However, such doubtful deviations must need a reliable diagnosis method(s) that capable of distinguishing slowgrowing preneoplastic lesions or benign lesions (14). Recently, the human serum has been broadly considered in the expedition for cancer biomarkers as blood is considered to deliver a dynamic representation of an individual's pathophysiological condition.

Neuron-specific enolase (NSE) and lactate dehydrogenase $(\mathrm{LDH})$ and have been acknowledged as prognosis markers for the SCLC (82). Contrarily, cytokeratin 19 fragment (CYFRA 21-1) has been identified as an independent biological factor for the NSCLC. Analyzing metadata of 2063 patients, CYFRA 21-1 was later corroborated as a possible biomarker for the diagnosis of NSCLC (83). Several antibody-based markers, such as carcinoembryonic antigen (CEA), cytokeratin 19 fragments (CYFRA21-1), carbohydrate antigen (CA) 125 and CA19, oncogene or tumor suppressor genes (e.g., Rb, p53, Bcl2), proliferation markers (KI67), angiogenesis markers (vascular endothelial growth factor, VEGF), and different antigens (e.g., NSE, CA125, tissue polypeptide antigen, etc.) have been explored, but still needs sufficient proof to be recognized as establishing markers for the diagnosis of LC (84). Therefore, a very few prognosis markers have been examined to clinical trials as most of these biomolecules had low accuracy and specificity to diagnose LC in early-stage. However, recent research suggested that the antibodies against tumor-associated antigens (TAAs), called tumor-associated autoantibodies (TAAbs), could be a possible pathway to early detection of LC (85). Compared with these traditional LC serological markers, TAAbs offer exceptional advantages regarding the early diagnosis of LC, even in some cases 5 years ahead of regular diagnosis.

\section{Bronchoscopy}

Bronchoscopy is an invasive procedure to examine different conditions of the lungs by inserting a camera-connected lighted tube (Bronchoscope) into the lungs through a patient's nose or mouth. As the inner tissues, bronchi, and bronchioles are exposed to the Bronchoscope, and it is possible to detect different anomalies in the lungs, including tumors, a sign of infection, excess mucus, etc. using bronchoscopy. There are two types of bronchoscopyflexible bronchoscopy and rigid bronchoscopy. Rigid bronchoscopy employs a piece of larger and rigid equipment, which provides access to the proximal airways. It is generally performed for the removal of foreign substances and airway stents to control massive hemoptysis. It is also used for tumor debulking and dilation of the airways (86). 

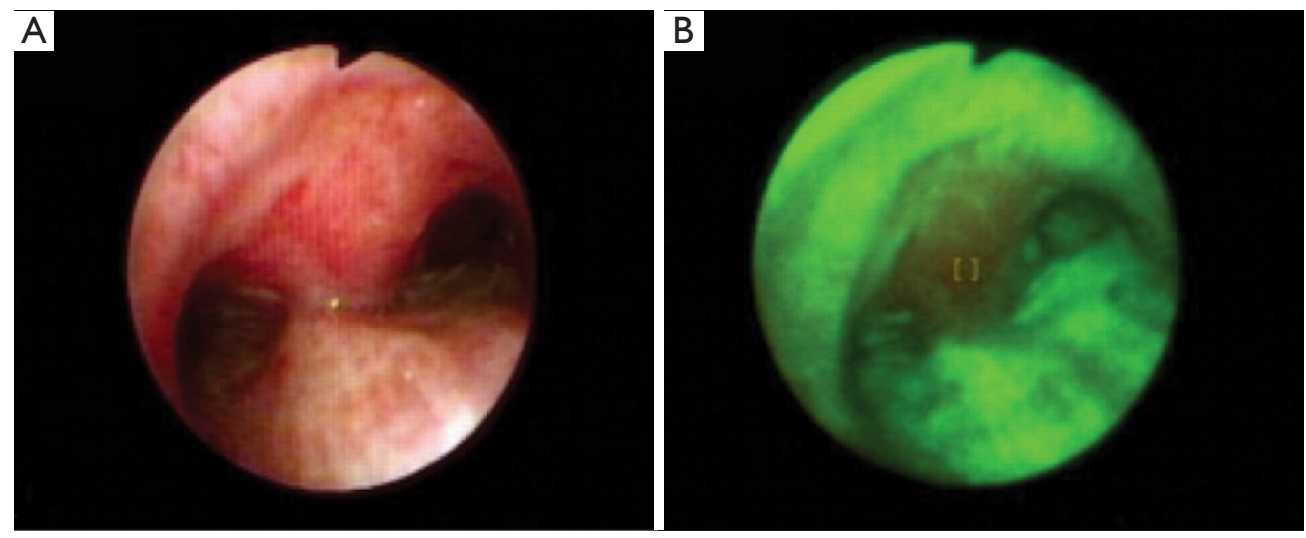

Figure 5 Different bronchoscopy procedures to identify LC in the same patient. (A) White-light bronchoscopy; (B) autofluorescence bronchoscopy image.

Contrarily, the flexible bronchoscopy uses a smaller and more flexible piece of equipment to access lower airways (e.g., third order of bronchi).

As bronchoscopy gives a detailed overview of different features of the lungs from inside, it is widely recommended for the diagnosis of early-stage cancer detection (87). In practice, fiber-optic bronchoscopy is widely used as it is safe $(0.12 \%$ complication rate) and easy to perform (88). Tofolean et al. summarized the importance of bronchoscopy in early LC detection by comparing the performance of different bronchoscopy procedures with several traditional approaches such as biopsy, X-ray, spiral CT, etc. (89). In this study, researchers demonstrated that bronchial brush procedures in bronchoscopy could achieve positive results in $88-94 \%$ cases, which is higher than bronchial aspiration and has minimum complications than bronchial biopsy (90). De Roza et al. conducted a study among 226 patients to determine the success rate of bronchoscopy to detect earlystage LC (91). Among 226 patients, $84.5 \%$ of patients were successfully diagnosed with LC using bronchoscopy. However, the chance of false-negative is still high in flexible bronchoscopy, as small tumors inside the tissues cannot be detected in this procedure. Recent advancements and researches have addressed this shortcoming and proposed different techniques to improve the accuracy of cancer detection using bronchoscopy. For example, Herth et al. proposed narrow-band imaging (NBI) in flexible bronchoscopy procedures to improve the sensitivity and specificity of detecting early-stage LC (92). They performed the study among 62 patients and found that NBI can lower the sensitivity without impacting the accuracy of cancer detection using bronchoscopy. Among different bronchoscopy approaches, autofluorescence bronchoscopy and white light bronchoscopy are more prominent in practice.

Autofluorescence bronchoscopy (AFB) is a type of bronchoscopic procedure where flexible bronchoscopy is performed with a blue light instead of a white light (93). The main reason to use blue light is to differentiate the normal tissue and the malignant tissue by color without using any fluorescence-enhancing drug. AFB uses the same equipment used in regular flexible bronchoscopy except for a dedicated endoscopic system for capturing blue light imaging. As the captured images are of different wavelengths, in $\mathrm{AFB}$, the normal tissues are visualized as green, and abnormal tissues are visualized as a reddishbrown color. For further inspection, normal bronchoscopic procedures are followed.

Compared to white light bronchoscopy (WLB), as shown in Figure 5, AFB offers low specificity in detecting early LC (94). Häussinger et al. performed a comparison study between AFB and WLB among 1,173 patients in Europe (95). Their research showed that sensitivity and specificity can be improved by combining WLB and AFB concerning WLB alone. In another study, Ueno et al. performed AFB among 31 patients to detect preinvasive bronchial lesions (early stage of invasive cancer) (96). Their results showed that AFB detected 64 lesions in the patients with a sensitivity of $94.7 \%$, which is higher than the WLB (73.7\%). In a recent study, Tremblay et al. performed an early cancer detection study among 1300 participants using AFB and low-dose CT scans (97). Their research showed that adding AFB and CT scan, it is possible to detect early cancer stages among high-risk 
patients. In conclusion, using AFB with other existing methods, such as WLB or CT scans, can improve earlystage LC detection significantly.

\section{Endobronchial ultrasound (EBUS)}

EBUS is an effective procedure with a minimum invasion procedure to diagnose different lung diseases. EBUS uses ultrasound with regular bronchoscopy to visualize the inner tissues and airwall of the lungs (98). Due to low risk and high diagnostic value, EBUS is highly recommended in routine practice and early detection of lung diseases. There are two systems available for EBUS-linear EBUS and radial EBUS (88). For linear EBUS, the ultrasound transducer is used in the distal end, utilizing a fixed array to visualize the curvilinear pattern of the airways. In radial EBUS, a mechanical radial mini probe is used to visualize the properties of the peripheral of the lungs.

In recent years, researchers have studied the effectiveness of EBUS in detecting early-stage LC among patients. Herth et al. conducted a study among 648 patients to demonstrate the feasibility of EBUS in cancer detection (99). Their results showed that EBUS could improve the results of the regular bronchoscopic procedure without any complications in the patient's conditions. In a recent study, the same group researchers observed 105 different cases where patients have centrally located tumors in the lungs (100). EBUS methods detected all the cases with sensitivity and specificity of $89 \%$ and $100 \%$, respectively. Miyazu et al. reported the effectiveness of EBUS in detecting early-stage LC in 16 different cases (101). Among these cases, EBUS has correctly diagnosed the depth of invasion. In another study, Takahashi et al. Used EBUS in detecting the degree of carcinoma invasion and found a $100 \%$ success rate in 14 cases (102). In conclusion, EBUS is proven as a highly effective procedure along with regular bronchoscopy to detect the depth and degree of invasion in the early stage of LC.

\section{Confocal endoscopy}

Confocal Endoscopy or confocal laser endoscopy (CLE) is an advanced imaging technique that permits real-time capturing of in vivo pictures of cellular and subcellular structures in the mucosa or living cells in lung tissue. CLE is a potential tool to explore alveolar elastic fiber structures, bronchus mucous membranes, and micro-vessels in the lung tissue. Hassan et al. studied 48 patients for the feasibility of CLE having with thinner probes to evaluate the malignancy of solitary pulmonary nodules. They employed r-EBUS coupled with CLE towards achieving $79.2 \%$ of accuracy (103). Fuchs et al. demonstrated a comprehensive study of 32 suspected patients who underwent CLE and bronchoscopy together. Their exploration showed significantly improved performance (sensitivity $96.0 \%$, specificity $87.1 \%$, accuracy $91.0 \%$ ) to diagnose neoplastic changes (104). Su et al. verified needle-based CLE (nCLE) to compare with CT, EBUS, and X-ray, respectively (105). Their case studies exhibited that nCLE was viable to entree the extraluminal pulmonary nodules through a microneedle for investigation with $91 \%$ accuracy. Comino et al. explored probe-based CLE (pCLE), integrated with computer-aided diagnoses (CAD) tools for the reliable identification of LC (106). Their structural graph analysis and computation methods were employed to deep-learning feature spaces to obtain enhanced CLE images (83.4\% accuracy), which contain adequate visual information of neoplastic cell patterns of lung tissue.

\section{Future trends and conclusion}

Even with new technologies and treatments, LC is one of the major causes of patient mortality in the world. Several research studies showed that LC can be treated successfully if diagnosed at an early stage. One possible future solution to increase LC detection at an early stage is the use of artificial intelligence and machine learning. The available medical metadata of LC patients and treatments can be accurately visualize using different machine learning approaches (107). Furthermore, artificial intelligence can also be utilized to analyze patients' behavior, lifestyle, and food habits to detect possible LC patients at an early stage (107-109). Additionally, medical data from different patients can be analyzed using machine learning and artificial intelligence to propose a better treatment plan.

\section{Acknowledgments}

Funding: None.

\section{Footnote}

Provenance and Peer Review: This article was commissioned by the Guest Editors (Roberto Gasparri \& Giulia Sedda) for the series "A New Era in Lung Cancer Care: from Early Diagnosis to Personalized Treatment", published in 
Shanghai Chest. This article has undergone external peer review.

Conflicts of Interest: All authors have completed the ICMJE uniform disclosure form (available at http://dx.doi. org/10.21037/shc.2020.03.03). The series "A New Era in Lung Cancer Care: from Early Diagnosis to Personalized Treatment" was commissioned by the editorial office without any funding or sponsorship. The authors have no other conflicts of interest to declare.

Ethical Statement: The authors are accountable for all aspects of the work in ensuring that questions related to the accuracy or integrity of any part of the work are appropriately investigated and resolved.

Open Access Statement: This is an Open Access article distributed in accordance with the Creative Commons Attribution-NonCommercial-NoDerivs 4.0 International License (CC BY-NC-ND 4.0), which permits the noncommercial replication and distribution of the article with the strict proviso that no changes or edits are made and the original work is properly cited (including links to both the formal publication through the relevant DOI and the license). See: https://creativecommons.org/licenses/by-nc-nd/4.0/.

\section{References}

1. World Health Organisation (press release), Latest global cancer data: Cancer burden rises to 18.1 million new cases and 9.6 million cancer deaths in 2018. [published 12 September 2018]. Available online: https://www.who.int/ cancer/PRGlobocanFinal.pdf

2. Dela Cruz CS, Tanoue LT, Matthay RA. Lung Cancer: Epidemiology, etiology, and prevention. Clin Chest Med 2011;32:605-44.

3. Borczuk AC. Uncommon types of lung carcinoma with mixed histology sarcomatoid carcinoma, adenosquamous carcinoma, and mucoepidermoid carcinoma. Arch Pathol Lab Med 2018;142:914-21.

4. Zhang Z, Wang Y, Zhao Q, et al. Mixed adenocarcinoma, sarcomatoid carcinoma and adenosquamous carcinoma of the prostate: A case report. Oncol Lett 2014;8:2325-7.

5. Walser T, Cui X, Yanagawa J, et al. Smoking and lung cancer: The role of inflammation. Proc Am Thorac Soc 2008;5:811-5.

6. Gaissert HA, Grillo HC, Shadmehr MB, et al. Uncommon Primary Tracheal Tumors. Ann Thorac Surg 2006;82:268-
72; discussion 272-3.

7. Wu S, Zhu W, Thompson P, et al. Evaluating intrinsic and non-intrinsic cancer risk factors. Nat Commun 2018;9:3490.

8. Kubik, A., Zatloukal P, Tomasek L, et al. A casecontrol study of lifestyle and lung cancer associations by histological types. Neoplasma 2008;55:192-9.

9. Song C, Li J, Liu Y, et al. Associations Between Hepatitis B Virus Infection and Risk of All Cancer Types. JAMA Network Open 2019;2:e195718.

10. Prasad R, Husain N, Karmakar S, et al. Lung cancer and Hepatitis C virus. South Asian J Cancer 2013;2:86.

11. Morales-Sánchez A, Fuentes-Pananá EM. Human viruses and cancer. Viruses 2014;6:4047-79.

12. Khan N, Afaq F, Mukhtar H. Lifestyle as risk factor for cancer: Evidence from human studies. Cancer Lett 2010;293:133-43.

13. Midthun DE. Early detection of lung cancer. F1000Res 2016;5. doi: 10.12688/f1000research.7313.1.

14. Blandin Knight S, Crosbie PA, Balata H et al. Progress and prospects of early detection in lung cancer. Open Biol 2017;7:1-12.

15. Subha B. Basu. Is Lung Cancer better detected using an X-Ray or CT Scan? 27 June 2006. Available online: https://www.itnonline.com/article/lung-cancer-betterdetected-using-x-ray-or-ct-scan

16. Lee H, Chen YP. Image based computer aided diagnosis system for cancer detection. Expert Syst Appl 2015;42:5356-65.

17. Wu D, Erwin D, Rosner GL. Sojourn time and lead time projection in lung cancer screening. Lung Cancer 2011;72:322-6.

18. Blanchon T, Bréchot JM, Grenier PA, et al. Baseline results of the Depiscan study: a French randomized pilot trial of lung cancer screening comparing low dose CT scan (LDCT) and chest X-ray (CXR). Lung Cancer 2007;58:50-8.

19. Gohagan JK, Marcus PM, Fagerstrom RM, et al. Final results of the Lung Screening Study, a randomized feasibility study of spiral CT versus chest $\mathrm{X}$-ray screening for lung cancer. Lung Cancer 2005;47:9-15.

20. Gordienko Y, Gang P, Hui J, et al. Deep learning with lung segmentation and bone shadow exclusion techniques for chest $x$-ray analysis of lung cancer. In: Hu Z, Petoukhov Z, Dychka $S$, et al. editors. Advances in Computer Science for Engineering and Education. Cham: Springer International Publishing AG, 2018;638-47.

21. Emory Healthcare, What Is Lung CT Screening \& How 
Does It Work? 2018. Available online: https://www. emoryhealthcare.org/centers-programs/lung-ct-program/ about.html

22. Saghir Z, Dirksen A, Ashraf H, et al. CT screening for lung cancer brings forward early disease. The randomized Danish Lung Cancer Screening Trial: status after five annual screening rounds with low-dose CT. Thorax 2012;67:296-301.

23. Henschke CI, Yankelevitz DF, Libby DM. Survival of patients with stage I lung cancer detected on CT screening. N Engl J Med 2006;355:1763-71.

24. Binson VA, Subramoniam M. Advances in Early Lung Cancer Detection: A Systematic Review. In: 2018 International Conference on Circuits and Systems in Digital Enterprise Technology (ICCSDET), 2018;1-5.

25. Makaju S, Prasad PWC, Alsadoon A, et al. Lung cancer detection using CT scan images. Procedia Computer Science 2018;125:107-14.

26. Tarro G, Paolini M, Rossi A. Molecular biology of lung cancer and future perspectives for screening. Available online: https://www.intechopen.com/books/massspectrometry-future-perceptions-and-applications/ molecular-biology-of-lung-cancer-and-futureperspectives-for-screening

27. Luo W, Rao M, Qu J, et al. Applications of liquid biopsy in lung cancer-diagnosis, prognosis prediction, and disease monitoring. Am J Transl Res 2018;10:3911-23.

28. Hofman P. Liquid biopsy for early detection of lung cancer. Curr Opin Oncol 2017;29:73-8.

29. Santarpia M, Liguori A, D'Aveni A, et al. Liquid biopsy for lung cancer early detection. J Thorac Dis 2018;10:S882-97.

30. Garzon R, Calin GA, Croce CM. MicroRNAs in cancer. Annu Rev Med 2009;60:167-79.

31. Wang H, Peng R, Wang J, et al. Circulating microRNAs as potential cancer biomarkers: the advantage and disadvantage. Clin Epigenetics 2018;10:59.

32. Yu H, Guan Z, Cuk K, et al. Circulating microRNA biomarkers for lung cancer detection in Western populations. Cancer meDdicine 2018;7:4849-62.

33. Bracken CP, Scott HS, Goodall GJ. A network-biology perspective of microRNA function and dysfunction in cancer. Nat Rev Genet 2016;17:719-32.

34. Zhu Y, Xiong K, Shi J, et al. A potential role of microRNAs in protein accumulation in cellular senescence analyzed by bioinformatics. PLoS One 2017;12:e179034.

35. Lin S, Gregory RI. MicroRNA biogenesis pathways in cancer. Nat Rev Cancer 2015;15:321-33.
36. Calin GA, Croce CM. MicroRNA signatures in human cancers. Nat Rev Cancer 2006;6:857-66.

37. Rosenfeld N, Aharonov R, Meiri E, et al. MicroRNAs accurately identify cancer tissue origin. Nat Biotechnol 2008;26:462.

38. Wozniak MB, Scelo G, Muller DC, et al. Circulating microRNAs as non-invasive biomarkers for early detection of non-small-cell lung cancer. PLoS One 2015;10:e0125026.

39. Boeri M, Verri C, Conte D, et al. MicroRNA signatures in tissues and plasma predict development and prognosis of computed tomography detected lung cancer. Proc Natl Acad Sci U S A 2011;108:3713-8.

40. Sozzi G, Boeri M, Rossi M, et al. Clinical utility of a plasma-based miRNA signature classifier within computed tomography lung cancer screening: a correlative MILD trial study. J Clin Oncol 2014;32:768-73.

41. Zaporozhchenko IA, Morozkin ES, Skvortsova TE, et al. Plasma miR-19b and miR-183 as potential biomarkers of lung cancer. PLoS One 2016;11:e165261.

42. Shen J, Todd NW, Zhang H, et al. Plasma microRNAs as potential biomarkers for non-small cell lung cancer. Lab Invest 2011;91:579-87.

43. Pattarayan D, Thimmulappa RK, Ravikumar V, et al. Diagnostic potential of extracellular MicroRNA in respiratory diseases. Clin Rev Allergy Immunol 2018;54:480-92.

44. Mestdagh P, Vandesompele J, Brusselle G, et al. Non-coding RNAs and respiratory disease. Thorax 2015;70:388-90.

45. Alipoor SD, Adcock IM, Garssen J, et al. The roles of miRNAs as potential biomarkers in lung diseases. Eur J Pharmacol 2016;791:395-404.

46. Noren Hooten N, Fitzpatrick M, Wood WH, et al. Agerelated changes in microRNA levels in serum. Aging (Albany NY) 2013;5:725-40.

47. Badrnya S, Baumgartner R, Assinger A. Smoking alters circulating plasma microvesicle pattern and microRNA signatures. Thromb Haemost 2014;112:128-36.

48. Zheng XH, Cui C, Zhou XX, et al. Centrifugation: an important pre-analytic procedure that influences plasma microRNA quantification during blood processing. Chin J Cancer 2013;32:667-72.

49. Becker N, Lockwood CM. Pre-analytical variables in miRNA analysis. Clin Biochem 2013;46:861-8.

50. Foye C, Yan IK, David W, et al. Comparison of miRNA quantitation by Nanostring in serum and plasma samples. PLoS One 2017;12:e0189165.

51. Inage T, Nakajima T, Yoshino I, et al. Early lung cancer 
detection. Clinics in chest mediciDne. 2018;39:45-55.

52. Motherby H, Nicklaus S, Berg A, et al. Semiautomated monolayer preparation of bronchial secretions using AutoCyte PREP. Acta Cytol 1999;43:47-57.

53. Holiday DB, McLarty JW, Farley ML, et al. Sputum cytology within and across laboratories. A reliability study. Acta Cytol 1995;39:195-206.

54. Fontana RS, Sanderson DR, Taylor WF, et al. Early lung cancer detection: results of the initial (prevalence) radiologic and cytologic screening in the Mayo clinic study. Am Rev Respir Dis 1984;130:561-5.

55. Frost JK, Ball WC Jr, Levin ML, et al. Early lung cancer detection: results of the initial (prevalence) radiologic and cytologic screening in the Johns Hopkins study. Am Rev Respir Dis 1984;130:549-54.

56. Doria-Rose VP, Marcus PM, Szabo E, et al. Randomized controlled trials of the efficacy of lung cancer screening by sputum cytology revisited: a combined mortality analysis from the Johns Hopkins lung project and the Memorial Sloan-Kettering lung study. Cancer 2009;115:5007-17.

57. Guzmán L, Depix MS, Salinas AM, et al. Analysis of aberrant methylation on promoter sequences of tumor suppressor genes and total DNA in sputum samples: a promising tool for early detection of COPD and lung cancer in smokers. Diagn Pathol 2012;7:87.

58. Dong N, Shi L, Wang DC, et al. Role of epigenetics in lung cancer heterogeneity and clinical implication. Semin Cell Dev Biol 2017;64:18-25.

59. Liu D, Peng H, Sun Q, et al. The indirect efficacy comparison of DNA methylation in sputum for early screening and auxiliary detection of lung cancer: a metaanalysis. Int J Environ Res Public Health 2017;14:E679.

60. Hulbert A, Jusue-Torres I, Stark A, et al. Early detection of lung cancer using DNA promoter hypermethylation in plasma and sputum. Clin Cancer Res 2017;23:1998-2005.

61. Wang X, Ling L, Su H, et al. Aberrant methylation of genes in sputum samples as diagnostic biomarkers for nonsmall cell lung cancer: A meta-analysis. Asian Pac J Cancer Prev 2014;15:4467-74.

62. Shiga T, Wajima ZI, Inoue T, et al. Predicting difficult intubation in apparently normal patients: a meta-analysis of bedside screening test performance. Anesthesiology 2005;103:429-37.

63. Wang W, Li T, Wang X, et al. FAM19A4 is a novel cytokine ligand of formyl peptide receptor 1 (FPR1) and is able to promote the migration and phagocytosis of macrophages. Cell Mol Immunol 2015;12:615-24.

64. Yan $W, X u$ N, Han X, et al. The clinicopathological significance of FHIT hypermethylation in non-small cell lung cancer, a meta-analysis and literature review. Sci Rep 2016;6:19303.

65. Fong KM, Biesterveld EJ, Virmani A, et al. FHIT and FRA3B 3p14. 2 allele loss are common in lung cancer and preneoplastic bronchial lesions and are associated with cancer related FHIT cDNA splicing aberrations. Cancer Res 1997;57:2256-67.

66. Gu C, Lu J, Cui T, et al. Association between MGMT promoter methylation and non-small cell lung cancer: a meta-analysis. PLoS One 2013;8:e72633.

67. Horvath G, Andersson H, Paulsson G. Characteristic odour in the blood reveals ovarian carcinoma. BMC Cancer 2010;10:643.

68. Matsumura K, Opiekun M, Oka H, et al. Urinary volatile compounds as biomarkers for lung cancer: a proof of principle study using odor signatures in mouse models of lung cancer. PLoS One 2010;5:e8819.

69. Liu H, Wang H, Li C, et al. Investigation of volatile organic metabolites in lung cancer pleural effusions by solid-phase microextraction and gas chromatography/mass spectrometry. J Chromatogr B Analyt Technol Biomed Life Sci 2014;945-946:53-9.

70. Brattoli M, Cisternino E, Dambruoso PR, et al. Gas Chromatography analysis with olfactometric detection (GC-O) as a useful methodology for chemical characterization of odorous compounds. Sensors (Basel) 2013;13:16759-800.

71. Lindinger $W$, Jordan A. Proton-transfer-reaction mass spectrometry (PTR-MS): On-line monitoring of volatile organic compounds at pptv levels. Chem Soc Rev 1998;27:347.

72. Spanĕl P, Smith D. Selected Ion Flow Tube mass spectrometry for on-line trace gas analysis in biology and medicine. Eur J Mass Spectrom (Chichester) 2007;13:77-82.

73. Wang C, Sahay P. Breath analysis using laser spectroscopic techniques: breath biomarkers, spectral fingerprints, and detection limits. Sensors 2009;9:8230-62.

74. Gloess AN, Yeretzian C, Knochenmuss R, et al. On-line analysis of coffee roasting with ion mobility spectrometrymass spectrometry (IMS-MS). Int J Mass Spectrom 2018;424:49-57.

75. Alam F, Jalal AH, Pala N. Selective detection of alcohol through ethyl-glucuronide (EtG) immunosensor based on 2D zinc oxide nanostructures. IEEE Sensors Journal 2019;19:3984-92.

76. Jalal AH, Umasankar Y, Ahmed A, et al. Towards wearable fuel cell sensor for transdermal monitoring of isoflurane - 
an anesthetic. Analytical Methods 2019;11:2007-12.

77. Devkota J, Ohodnicki PR, Greve DW. SAW sensors for chemical vapors and gases. Sensors (Basel) 2017;17:1-28.

78. Semwal V, Shrivastav AM, Verma R, et al. Surface plasmon resonance based fiber optic ethanol sensor using layers of silver/silicon/hydrogel entrapped with $\mathrm{ADH} / \mathrm{NAD}$. Sensors Actuators B Chem 2016;230:485-92.

79. Obermeier J, Trefz P, Wex K, et al. electrochemical sensor system for breath analysis of aldehydes, CO and NO. J Breath Res 2015;9:016008.

80. Mazzone P J, Wang X, Xu Y, et al. Exhaled breath analysis with a colorimetric sensor array for the identification and characterization of lung cancer. J Thorac Oncol 2012;7:137-42.

81. Machado R F, Laskowski D, Deffenderfer O, et al. Detection of lung cancer by sensor array analyses of exhaled breath. Am J Respir Crit Care Med 2005;171:1286-91.

82. Liu $X$, Zhang $W$, Yin $W$. The prognostic value of the serum neuron specific enolase and lactate dehydrogenase in small cell lung cancer patients receiving first-line platinum-based chemotherapy. Medicine (Baltimore) 2017;96:e8258.

83. Xu Y, Xu L, Qiu M. Prognostic value of serum cytokeratin 19 fragments (Cyfra 21-1) in patients with non-small cell lung cancer. Sci Rep 2015;5:9444.

84. Solassol J, Maudelonde T, Mange A. Clinical relevance of autoantibody detection in lung cancer. J Thorac Oncol 2011;6:955-62.

85. Yang B, Li X, Ren T. Autoantibodies as diagnostic biomarkers for lung cancer: A systematic review. Cell Death Discovery 2019;5:126.

86. Islam S, Colt HG, Finlay G. Flexible bronchoscopy in adults: Preparation, procedural technique, and complications. (2016).

87. Kamath AV, Chhajed PN. Role of bronchoscopy in early diagnosis of lung cancer. Indian J Chest Dis Allied Sci 2006; 48:265.

88. Herth FJ. Bronchoscopic techniques in diagnosis and staging of lung cancer. Breathe 2011;7:324-37.

89. Tofolean DE, Fildan AP, Dantes E, et al. The Importance of Bronchoscopy in Early Lung Cancer (LC) Diagnosis. ARS Medica Tomitana 2016;22:270-7.

90. Popp W, Rauscher H, Ritschka L, et al. Diagnostic sensitivity of different techniques in the diagnosis of lung tumors with the flexible fiberoptic bronchoscope. Comparison of brush biopsy, imprint cytology of forceps biopsy, and histology of forceps biopsy. Cancer 1991;67:72-5.

91. De Roza MA, Quah KH, Tay CK, et al. Diagnosis of peripheral lung lesions via conventional flexible bronchoscopy with multiplanar CT planning. Pulm Med 2016;2016:5048961.

92. Herth FJ, Eberhardt R, Anantham D et al. Narrowband imaging bronchoscopy increases the specificity of bronchoscopic early lung cancer detection. J Thorac Oncol 2009;4:1060-5.

93. Autofluorescence Bronchoscopy. Chest 2003;123:1701-2.

94. Zaric B, Perin B, Carapic V, et al. Diagnostic value of autofluorescence bronchoscopy in lung cancer. Thoracic Cancer 2013;4:1-8.

95. Häussinger K, Becker H, Stanzel F, et al. Autofluorescence bronchoscopy with white light bronchoscopy compared with white light bronchoscopy alone for the detection of precancerous lesions: a European randomised controlled multicentre trial. Thorax 2005;60:496-503.

96. Ueno K, Kusunoki Y, Imamura F, et al. Clinical experience with autofluorescence imaging system in patients with lung cancers and precancerous lesions. Respiration 2007;74:304-8.

97. Tremblay A, Taghizadeh N, McWilliams AM et al. Low prevalence of high-grade lesions detected with autofluorescence bronchoscopy in the setting of lung cancer screening in the pan-canadian lung cancer screening study. Chest 2016;150:1015-22.

98. UC San Diego Health. Endobronchial Ultrasound Bronchoscopy (EBUS). Published in 2018. Available online: https://health.ucsd.edu/specialties/pulmonary/ procedures/Pages/endobronchial.aspx

99. Herth F, Becker HD, Manegold C, et al. Endobronchial ultrasound (EBUS)-assessment of a new diagnostic tool in bronchoscopy for staging of lung cancer. Onkologie 2001;24:151-4.

100.Herth F, Becker HD, LoCicero J, et al. Endobronchial ultrasound in therapeutic bronchoscopy. Eur Respir J 2002;20:118-21.

101. Miyazu Y, Miyazawa T, Kurimoto N, et al. Endobronchial ultrasonography in the assessment of centrally located early-stage lung cancer before photodynamic therapy. American journal of respiratory and critical care medicine. 2002;165:832-7.

102. Takahashi H, Sagawa M, Sato M, et al. A prospective evaluation of transbronchial ultrasonography for assessment of depth of invasion in early bronchogenic squamous cell carcinoma. Lung Cancer 2003;42:43-9.

103. Hassan T, Thiberville L, Hermant C. Assessing the feasibility of confocal laser endomicroscopy in solitary pulmonary nodules for different part of the lungs, using either 0.6- or 1.4-mm probes. PLoS One 2017;12:e0189846. 
104.Fuchs F S, Zirlik S, Hildner K. Confocal laser endomicroscopy for diagnosing lung cancer in vivo. Eur Respir J 2013;41:1401-8.

105.Su Z, Zhong C, Li S. Needle-based confocal laser endomicroscopy in the diagnosis of peripheral pulmonary nodule: a preliminary report. J Thorac Dis 2017;9:2608-12.

106. Comino RMO, Gil D, Minchole E, et al. Classification of Confocal Endomicroscopy Patterns for Diagnosis of Lung Cancer. J Thorac Oncol 2017;12:S1889.

107.Jalal AH, Alam F, RoyChoudhury S, et al. Prospects and

doi: $10.21037 /$ shc.2020.03.03

Cite this article as: Jalal AH, Sikder AK, Alam F, Samin S, Rahman SS, Khan MMA, Siddiquee MR. Early diagnosis with alternative approaches: innovation in lung cancer care. Shanghai Chest 2021;5:7. challenges of volatile organic compound sensors in human healthcare. ACS Sensors 2018;3:1246-63.

108.Jalal AH, Umasankar Y, Christopher F, et al. A model for safe transport of critical patients in unmanned drones with a 'watch' style continuous anesthesia sensor. J Electrochemical Society 2018;165:B3071-7.

109. Siddiquee MR, Xue T, Marquez JS, et al. Sensor Fusion in Human Cyber Sensor System for Motion Artifact Removal from NIRS Signal. 2019. 12th International Conference on Human System Interaction (HSI) 2019;192-6. 